\title{
Synthetic Biogenesis of Bacterial Amyloid Nanomaterials with Tunable Inorganic-Organic Interfaces and Electrical Conductivity
}

\author{
Urartu Ozgur Safak Seker, ${ }^{\dagger, \ddagger}, \|$ Allen Y. Chen, ${ }^{\dagger, \ddagger}$ Robert J. Citorik, ${ }^{\dagger, \ddagger, \S}$ and Timothy K. Lu* ${ }^{* \dagger, \ddagger, \S}$
}

${ }^{\dagger}$ Department of Electrical Engineering and Computer Science, Massachusetts Institute of Technology, Cambridge, Massachusetts 02139, United States

${ }^{\ddagger}$ Department of Biological Engineering, Massachusetts Institute of Technology, Cambridge, Massachusetts 02139, United States

${ }^{\S}$ MIT Microbiology Program, 77 Massachusetts Avenue, Cambridge, Massachusetts 02139, United States

"UNAM-Institute of Materials Science and Nanotechnology, Bilkent University, 06800 Ankara, Turkey

Supporting Information

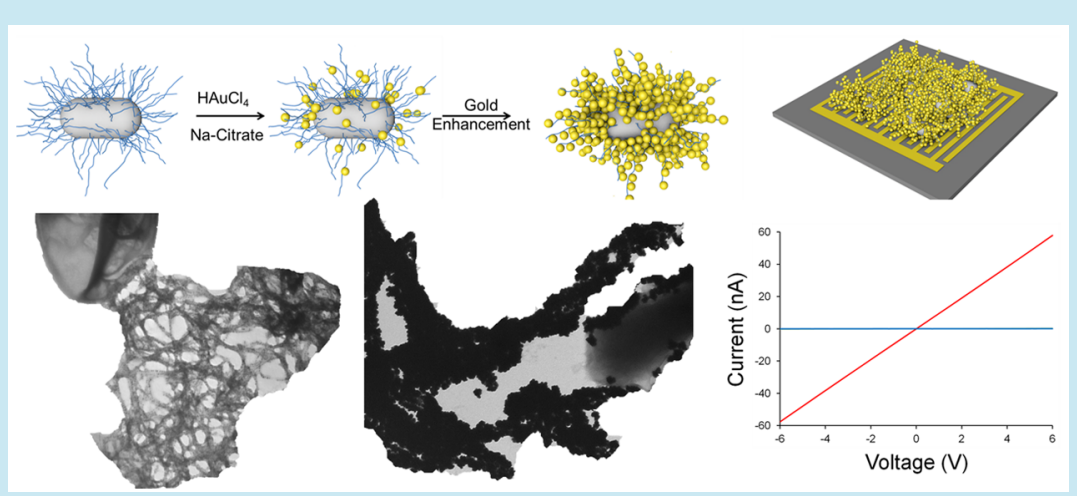

ABSTRACT: Amyloids are highly ordered, hierarchal protein nanoassemblies. Functional amyloids in bacterial biofilms, such as Escherichia coli curli fibers, are formed by the polymerization of monomeric proteins secreted into the extracellular space. Curli is synthesized by living cells, is primarily composed of the major curlin subunit CsgA, and forms biological nanofibers with high aspect ratios. Here, we explore the application of curli fibers for nanotechnology by engineering curli to mediate tunable biological interfaces with inorganic materials and to controllably form gold nanoparticles and gold nanowires. Specifically, we used cell-synthesized curli fibers as templates for nucleating and growing gold nanoparticles and showed that nanoparticle size could be modulated as a function of curli fiber gold-binding affinity. Furthermore, we demonstrated that gold nanoparticles can be preseeded onto curli fibers and followed by gold enhancement to form nanowires. Using these two approaches, we created artificial cellular systems that integrate inorganic-organic materials to achieve tunable electrical conductivity. We envision that cell-synthesized amyloid nanofibers will be useful for interfacing abiotic and biotic systems to create living functional materials..

KEYWORDS: biofilm proteins, functional amyloids, nanomaterial assembly, living materials

B acteria can build dynamic communities composed of living microbes and extracellular matrices, which can include materials such as polysaccharides, DNA, and amyloid proteins. $^{1,2}$ Though biofilms are often studied in the context of clinical infections, here we demonstrate that they can be used to construct biomaterials that are composed of living cells and extracellular materials, such as curli fibers. Recent studies have revealed insights into the self-assembly mechanisms which underlie curli fiber amyloid formation. ${ }^{3,4}$ Because of their distinctive molecular structures and self-assembly characteristics, curli fibers have the potential to be engineered for nanoscience and materials applications. 5 The amenability to genetic control ${ }^{6}$ and long-range and high-aspect-ratio characteristics of bacterial amyloid fibers should allow them to be useful nanotechnology tools. Here, we engineered curli amyloid nanofibers in living E. coli communities as templates for nanomaterial assembly and used them to mediate tunable electrical conductivity.

We first achieved tight genetic control over curli fiber assembly. Curli fibers are primarily composed of CsgA monomers that self-assemble on the cell surface. ${ }^{1}$ The endogenous cellular machinery for curli formation involves the $\operatorname{csg} B A C$ and $\operatorname{csg} D E F G$ operons. The $\operatorname{csg} B A C$ operon encodes the minor and major curlin subunit proteins CsgB and CsgA, respectively, as well as the accessory protein CsgC. CsgB is localized on the cell membrane and provides nucleation sites for the curlin monomer CsgA. ${ }^{1,7,8}$ The $\operatorname{csg} D E F G$ operon codes for CsgE, CsgF, and CsgG, required for the secretion and translocation of curli proteins into the extracellular space, in

Received: June 8, 2016

Published: October 30, 2016 

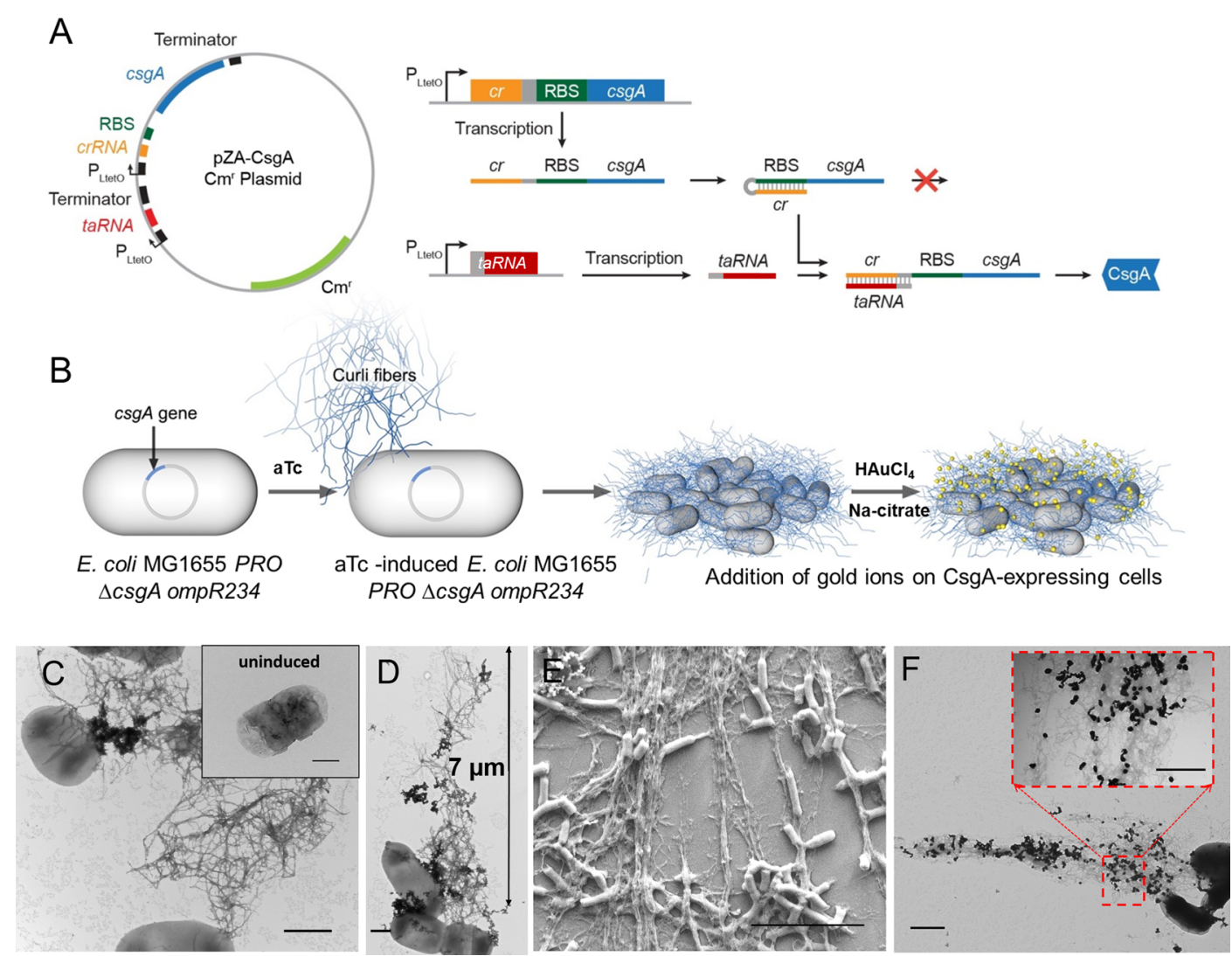

Figure 1. (A) Synthetic riboregulator circuit places $\operatorname{csg} A$ gene expression under the tight regulatory control of anhydrotetracycline (aTc). Transcription of $\operatorname{csg} A$ is controlled by a $\mathrm{P}_{\text {Lteto }}$ promoter, which is activated in the presence of aTc. Translation of $\operatorname{csg} A$ mRNA is repressed by a cisrepressing RNA ( $c r$ ), which sequesters the ribosome binding site (RBS) upstream of $\operatorname{csg} A$ to prevent the translation of any transcribed mRNAs. A trans-activating RNA (taRNA), which is also inducibly expressed by aTc, relieves repression by $c r$ and enables production of the CsgA protein and the formation of curli fibers. (B) Schematic of cell-based curli fibril formation using the riboregulated csgA circuitry along with controlled goldnanoparticle formation in the presence of sodium citrate and gold(III) chloride on curli fibers. (C and D) Long micron-scale curli fibers are synthesized when wild-type CsgA (CsgA-Wt) production is induced by aTc. The inset shows the absence of curli fiber formation in uninduced conditions. (E) Scanning electron microscopy image of cells induced by aTc to express curli fibers. (F) Gold-nanoparticle formation and assembly on cell-synthesized wild-type curli fibers composed of CsgA-Wt using $\mathrm{HAuCl}_{4}$ as the precursor material. The scale bar in part $\mathrm{E}$ represents $5 \mu \mathrm{m}$, while the other scale bars represent $500 \mathrm{~nm}$.

addition to the transcriptional regulator CsgD, which integrates multiple environmental signals to control curli synthesis.", 10

Since there is a complex endogenous network regulating the secretion of curli fibers in natural systems, ${ }^{1,6}$ we used a synthetic gene circuit from Chen et al. ${ }^{5}$ to implement externally inducible control of CsgA expression. In this circuit, $\operatorname{csg} A$ is under the control of an engineered riboregulator circuit such that its expression is tightly repressed in the absence of anhydrotetracycline $(\mathrm{aTc})$ and induced in the presence of $\mathrm{aTc}$ (Figure 1A). ${ }^{112}$ This gene circuit was transformed into an $E$. coli MG1655 PRO ompR234 host strain with the endogenous $\operatorname{csg} A$ gene removed $(\Delta \operatorname{csg} A))^{5}$ The ompR234 background confers the ability to assemble curli under liquid culture conditions by upregulating CsgD expression. ${ }^{15,17}$ This system enables the controlled formation of curli fibers and facile testing of $\operatorname{csg} A$ variants.

\section{RESULTS AND DISCUSSION}

We induced CsgA expression by growing bacterial cells at 30 ${ }^{\circ} \mathrm{C}$ on YESCA agar with aTc for $72 \mathrm{~h}$ or in liquid M63 minimal medium with aTc for $48 \mathrm{~h}$ (Figure 1C). Agar-grown curli samples were harvested and purified for biophysical characterization whereas liquid-grown curli were used as templates for nanogold assembly (see Methods). Using transmission electron microscopy (TEM), we verified that curli fibers assembled from the synthetic $\operatorname{csg} A$ construct were well ordered and assembled in long nanofibers with lengths into the micrometer range (Figure 1C-E). The scanning electron microscopy (SEM) image in Figure 1E depicts the morphology of E. coli expressing curli fibers.

We next explored whether cell-secreted curli fibers could be used as a nanomaterials assembly platform (Figure 1B). We added $50 \mu \mathrm{M} \mathrm{HAuCl}_{4}$ and $10 \mu \mathrm{M}$ sodium citrate to liquidgrown bacterial cells that synthesized curli fibers. Examination with TEM revealed the formation of $53 \pm 20 \mathrm{~nm}$ gold nanoparticles adhered to wild-type curli (CsgA-Wt) fibers on the outer cell surface of $E$. coli (Figure $1 \mathrm{~F}$ and Figure 3).

We hypothesized that the assembly of gold nanoparticles on curli fibers could be tuned by engineering the CsgA monomer to control the nucleation of gold seeds. Curli is formed by the assembly of monomeric CsgA subunits, and thus changes in the monomer should lead to alterations in the biochemical properties of the resulting curli fibers and their ability to bind gold and assemble gold nanomaterials. To test this hypothesis, we displayed heterologous inorganic-material-nucleating peptides $^{13,14}$ on curli fiber surfaces. This approach leverages previous work demonstrating that inorganic-material-binding 
A

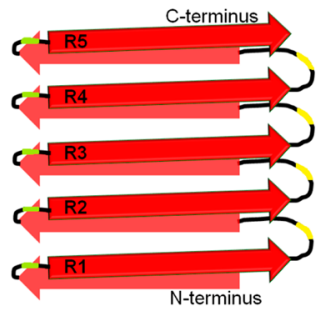

Sec $M K L L K V A A$ I A A I V F G S A $A$

N22 GVVPQYGGGGNHGGGGNNSGPN $\mathrm{N}^{\mathrm{L1}}$

R1 S E L I Y Y Y G G G N S A A L Q T D A R N

R2 $S D L T$ I T Q H G G $G_{G}^{44} N A D \vee G Q G S^{45} D$

R3 $S S I D L T Q R G F G N S A T L D Q W N G K N$

R4 $S$ E M T V K Q F G GG $G_{G}^{48} A A \vee D Q T A^{49} N$

R5 S S V N T $Q \vee G F^{4 / 10} N N A T A H Q Y^{411}$

B

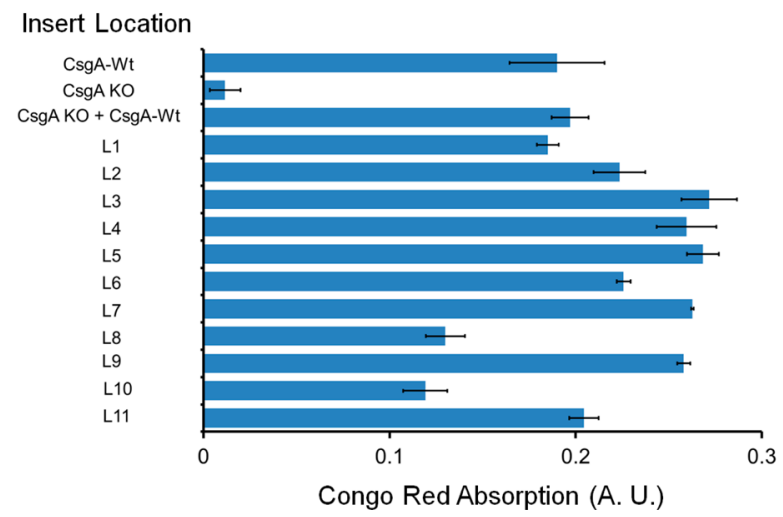

Figure 2. Assembly of the major curlin subunit CsgA into curli is robust to peptide insertions at various locations within its sequence. (A) Positions at which $7 \mathrm{xHis}$ peptides were inserted are labeled in the CsgA protein sequence (triangles, L1-L11). (B) The ability of cell-secreted CsgA variants to form curli amyloid fibers was quantified by a liquid Congo red assay. Congo red absorptions are given in arbitrary units (A.U.). CsgA-Wt represents curli samples produced by E. coli BW25113. CsgA KO represents the E. coli BW25113 $\Delta$ csgA strain, which does not contain any CsgAexpressing plasmids. CsgA KO + CsgA-Wt is the E. coli BW25113 $\Delta$ csgA strain transformed with the CsgA-Wt-expressing plasmid. All other strains (L1-L11) are E. coli BW25113 $\Delta$ csgA transformed with plasmids expressing CsgA with various 7xHis peptide insertions. Insertions were made at the following sites in the CsgA peptide sequence: L1: after N42, L2: after G52, L3: after D62, L4: after G75, L5: after S85, L6: after F97, L7: after N107, L8: after G120, L9: after A130, L10: after F142, and L11: after the C-terminal Y151. Three independent samples were prepared and measured. Error bars indicate standard deviation.

peptide motifs can modulate the growth and characteristics of inorganic materials. $^{15,16}$

As a prerequisite to displaying peptides on curli fibers to modulate gold binding, we first mapped out locations within the CsgA protein that tolerate the insertion of foreign peptides using a histidine heptamer (7xHis). In a putative model structure of CsgA, each repeat unit in the peptide sequence (denoted R1, R2, R3, R4, R5) forms two $\beta$ strands linked by a flexible loop, and repeats are, in turn, linked to each other by flexible loops. ${ }^{6}$ We previously inserted histidine heptamers at the C-terminus of the CsgA peptide sequence and now further explored the effect of such insertions at the $\mathrm{N}$-terminus of the first $\beta$ strand as well as in all of the flexible loops between $\beta$ strands (Figure 2A). The CsgA variants were tested for their ability to form amyloid fibrils by a liquid Congo Red (CR) binding assay. ${ }^{8}$ In all instances, the CsgA variants demonstrated effective binding of $\mathrm{CR}$ (Figure $2 \mathrm{~B}$ ). Based on these results, we fused a histidine heptamer to the C-terminus of CsgA (hereafter referred to as CsgA-P1). In addition, we fused gold-binding peptides to the C-terminus of CsgA, including LKAHLPPSRLPS (P2), ${ }^{17}$ AYSSGAPPMPPF (P3), ${ }^{18}$ and TGTSVLIATPGV (P4) ${ }^{19}$ to create CsgA-P2, CsgA-P3, and CsgA-P4, respectively.

To explore the functional and structural effects of these peptide fusions, we analyzed the gold-binding affinities and secondary structures of wild-type and engineered curli fibers. We purified curli fibers ${ }^{20}$ and used quartz crystal microbalancedissipation (QCM-D) to study the affinity of curli fibers for gold-coated sensor surfaces. ${ }^{1,21}$ Interaction isotherms of engineered curli fibers with the gold surface were used to calculate gold-binding affinity constants with a single-step Langmuir adsorption model (Table 1). ${ }^{22}$ Raw data for curli

Table 1. Affinity Binding Constants $\left(K_{\mathrm{D}}\right)$ between Gold Surfaces with Curli Fiber Variants and Secondary-Structure Composition of Curli Fiber Variants ${ }^{a}$

\begin{tabular}{cccccc} 
& & \multicolumn{4}{c}{ Secondary Structure Elements $(\%)$} \\
\cline { 3 - 6 } Curli Variants & $\boldsymbol{K}_{\mathbf{D}}(\boldsymbol{\mu} \mathbf{M})$ & Helical & Strand & Turn & Coil \\
CsgA-Wt & $22.5 \pm 3.73$ & 5.1 & 41.5 & 21.6 & 31.9 \\
CsgA-P1 & $2.5 \pm 0.28$ & 4.2 & 41.1 & 21.5 & 33.2 \\
CsgA-P2 & $1.2 \pm 0.35$ & 5.4 & 42.9 & 21.1 & 30.6 \\
CsgA-P3 & $0.32 \pm 0.03$ & 6.1 & 39.3 & 21.8 & 32.8 \\
CsgA-P4 & $0.65 \pm 0.25$ & 14.5 & 32.7 & 21.4 & 31.3
\end{tabular}

${ }^{a}$ Binding constants were determined using adsorption data gathered from QCM-D experimental data (Figure S1). The percentages of secondary-structure elements for the respective curli fibers were calculated using CDPro with the raw data from CD experiments (Figure S2).

adsorption and the model fitted to frequency shifts resulting from curli adsorption, as a function of curli concentration, are given in Figure S1. The gold-binding affinity constant for CsgAWt was found to be $22.5 \pm 3.73 \mu \mathrm{M}$. The insertion of $7 \mathrm{xHis}$ into CsgA increased the affinity of the resulting CsgA-P1 curli fibers to the gold surface by $\sim 9$-fold $(2.5 \pm 0.28 \mu \mathrm{M})$. Enhanced binding affinities were also observed for the CsgAgold binding peptide fusions. Compared to CsgA-Wt, CsgA-P2, CsgA-P3, and CsgA-P4 exhibited 20-fold, 70-fold, and 34fold increases in gold-binding affinities, respectively. 
Peptide Name

P1

$\mathrm{P} 2$

P3

P4
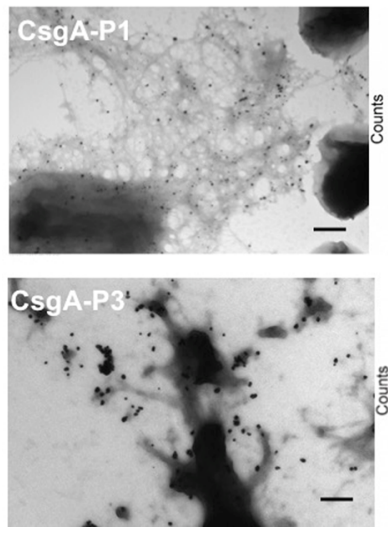

Sequence

$\mathrm{HHHHHHH}$

LKAHLPPSRLPS

AYSSGAPPMPPF TGTSVLIATPGV
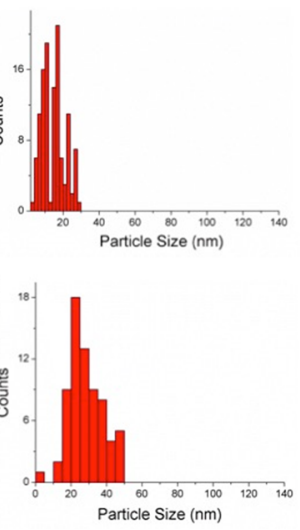
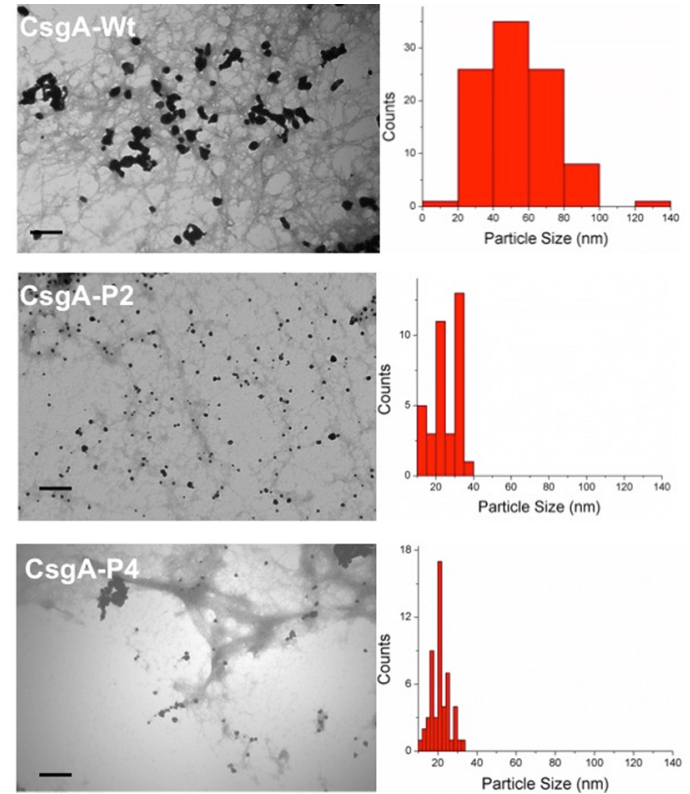

Figure 3. Representative TEM images of gold nanoparticles nucleated on peptide-displaying curli fibers synthesized by genetically engineered E. coli. Histograms describing the nanoparticle size distribution are given along with each representative image (80-90 nanoparticles were analyzed for each variant from multiple images). Scale bars in the images represent $100 \mathrm{~nm}$.

We next analyzed the secondary-structure motifs of CsgA-Wt and CsgA-peptide-fusion variants using circular dichroism (CD) spectroscopy, since molecular-binding events can potentially be impacted by structure. ${ }^{23,24} \mathrm{CD}$ data of the curli fibers were fit to a model to extract the percentages of the $\alpha$ helical, $\beta$ sheet, $\beta$ turn, and random coil motifs of CsgA-Wt and its peptide-fusion variants (Figure S2). The secondarystructure-motif contents of the curli variants are given in Table 1. Helical secondary structure motifs were the least represented in the secondary structures of the curli fibers examined, whereas sheet structures were the most represented. Fusing short peptide sequences with CsgA did not significantly change the distribution of secondary-structure motifs of the resulting curli fibers. $\beta$ strands form the $\beta$ sheet structure of amyloids. ${ }^{25}$ Secondary structure analysis (Table 1 ) found that the $\beta$ strand content was the dominant secondary structure element in all versions of the CsgA protein we tested, indicating that the amyloid structure was preserved. Consistent with these findings, YESCA agar-based CR assays of cells expressing CsgA$\mathrm{Wt}$ and the different curli fiber variants indicated that the curli variants maintained amyloid fiber features (Figure 2 and Figure S3). This indicates that curli can tolerate the insertion of these peptide sequences without significant detriment to structural integrity.

We then sought to demonstrate that cellular consortia producing peptide-displaying curli fibers can serve as tunable templates for nanoparticle synthesis. Gold-nanoparticle formation can be explained by a model in which gold ions are first reduced to metallic gold followed by formation of gold nuclei. Coalescence of gold nuclei then results in gold-nanoparticle formation. ${ }^{26}$ This effect can be modulated through peptide domains. For example, histidine has been shown to affect goldnanoparticle formation through interaction of the imidazole ring contained by histidine with gold ions. This interaction creates a coordination complex which ultimately influences gold-nanoparticle formation. ${ }^{27,28}$ A recent study on proteinmodulated gold-nanoparticle synthesis showed that $\mathrm{Au}(\mathrm{I})$ initially bound to the $\varepsilon$ - $\mathrm{N}$ of histidine and then spontaneously dissociated from histidine and transformed into $\mathrm{Au}(\mathrm{III})$ and $\mathrm{Au}(0) . \mathrm{Au}(0)$ accumulated on the protein as a function of time and led to the formation of gold nanoparticles from 2.9 to 16.9 $\mathrm{nm}$ in size. ${ }^{28}$

Gold-nanoparticle formation was initiated on curli variants produced by bacteria via the addition of $\mathrm{HAuCl}_{4}$ and sodium citrate to curli-displaying cells. Formation of gold nanoparticles in the presence of different curli variants resulted in nanoparticles with different sizes (Figure 3). With curli fibers composed of CsgA-P1, CsgA-P2, CsgA-P3, and CsgA-P4, the average sizes of the nanoparticles formed were $14 \pm 6 \mathrm{~nm}, 25 \pm$ $7.5 \mathrm{~nm}, 28 \pm 10 \mathrm{~nm}$, and $21 \pm 5 \mathrm{~nm}$, respectively. The gold nanoparticles nucleated by all of the curli variants were smaller in size than those nucleated by CsgA-Wt. This data indicates that heterologous peptides displayed on curli fibers can modulate the formation of gold nanoparticles.

One application of engineered cellular populations expressing functionalized curli fibers is to interface living biological cells with nonliving materials. These hybrid biotic-abiotic systems could combine the properties of living systems, which potentially include signal integration, environmental responsiveness, self-repair, and self-organization, ${ }^{29}$ with nonliving systems that confer functionalities such as electrical conductivity. We explored the use of curli fibers to create metallic nanowires on cell surfaces, which have potential bioenergy or bioelectronics applications. ${ }^{30,31}$ Here, we took two different approaches to create conductive nanowires using curli. In the first step, premade gold nanoparticles were used (either NiNTA coated or bare gold nanoparticles) to functionalize CsgAP1 through specific bioconjugation. Alternatively, gold nanoparticles were grown on peptide-fused curli fibers. In both cases, the resulting structures were gold-enhanced to build continuous conductive nanowires.

We initially used cell-synthesized CsgA-P1 fibers as a template for gold nanowire formation. In the first approach, premade nickel-nitrilotriacetic acid (NiNTA)-coated gold 


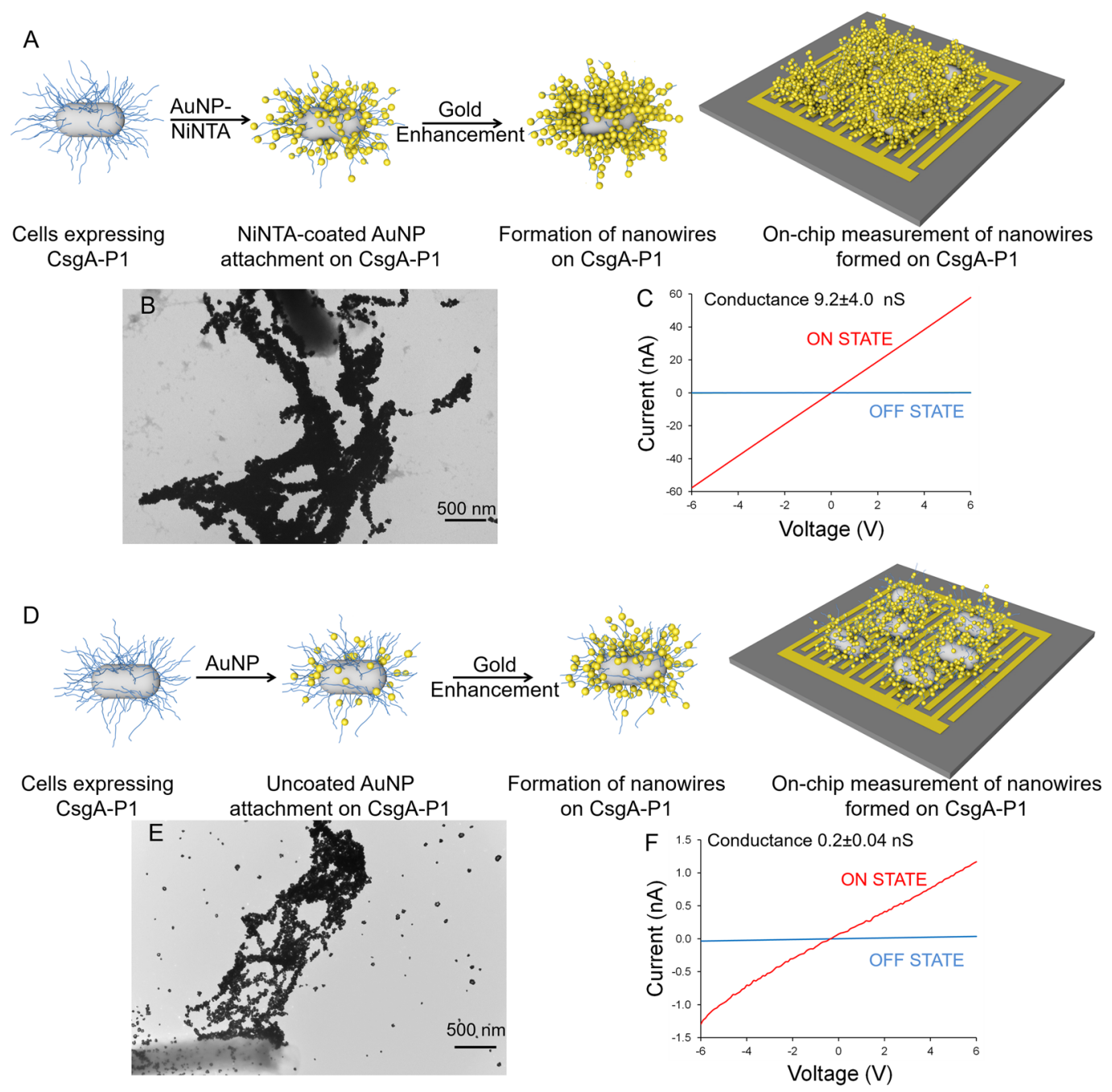

Figure 4. Conductive nanowire formation on cell-synthesized curli fibers with preformed gold nanoparticles followed by gold enhancement. (A-C) NiNTA-conjugated gold nanoparticles (AuNP-NiNTA) and (D-E) unconjugated gold particles (AuNP) were added to CsgA-P1-based curli fibers. Subsequent gold enhancement resulted in the formation of nanowires, as seen by electron microscopy (B and D). Conductance measurements on the cellular populations $(\mathrm{C}$ and $\mathrm{F})$ revealed a significant increase in conductance when curli-fiber-organized gold nanowires were induced by aTc (ON STATE) versus the uninduced state (OFF STATE). Conductance values are mean measurements from three independent samples from three electrodes. The images were collected from the samples scraped from the surface of the interdigitated electrodes used for measurements..

nanoparticles were targeted to the fibers based on interactions between NiNTA and the histidine residues of CsgA-P1 (Figure $4 \mathrm{~A})$. In the second approach, we used premade uncoated gold nanoparticles that bound to CsgA-P1 fibers under buffer conditions that allowed interactions between gold particles and curli in the absence of NiNTA (Figure 4D). In both cases, we then used gold enhancement on the premade gold nanoparticles that adhered to CsgA-P1-based curli fibers to create metallic gold nanowires attached to cell surfaces. The resulting gold nanowires templated on CsgA-P1 fibers, with a higher density obtained from the NiNTA-coated gold-nanoparticle chains (Figure 4B) compared to the unconjugated goldnanoparticle chains (Figure 4E).

To determine whether these nanowires could enable conductive cell populations, bacterial cultures were either uninduced or induced for CsgA-P1 curli expression with aTc, followed by gold attachment and enhancement, and tested for conductance using interdigitated electrodes (IDEs). Cells induced with aTc generated conductive networks composed of curli and gold, whereas cells that were uninduced exhibited minimal conductance (Figure 4C and 4F). The conductance of the nanowire networks formed with NiNTA-modified gold nanoparticles (Figure 4A-C) was greater than those formed with unmodified gold nanoparticles (Figure 4D-F), consistent with the relative densities of the nanowires (Figure $4 \mathrm{~B}$ and $4 \mathrm{E}$ ).

As an alternative to gold enhancement of preformed gold nanoparticles arrayed on curli nanofibers, we directly grew gold nanowires on curli fibers. We started with gold nanoparticles that were nucleated on the curli fiber variants shown in Figure 2 followed by gold enhancement. Conductance measurements were carried out on cell populations subjected to gold nucleation and enhancement. In all cases, induction of curli expression in cell populations with aTc resulted in significant increases in conductance above cell populations in the absence of aTc (Figure 5B-E). All the conductivities resulting from nucleated gold nanoparticles followed by gold enhancement (Figure 5) were reduced compared with the conductivities obtained from preformed gold nanoparticles followed by gold enhancement (Figure 4). This work demonstrates that inorganic-organic nanoscale systems templated by engineered 
A

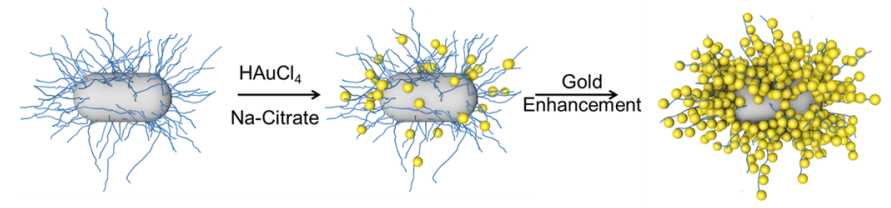

Cells expressing curli

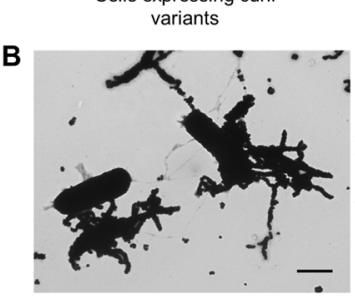
AuNP nucleation on
curli variants

Formation of nanowires on curli

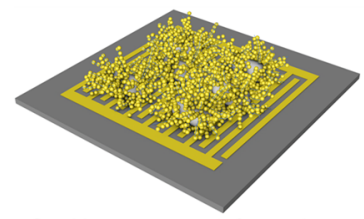

On-chip measurement of nanowire formed on curli variants
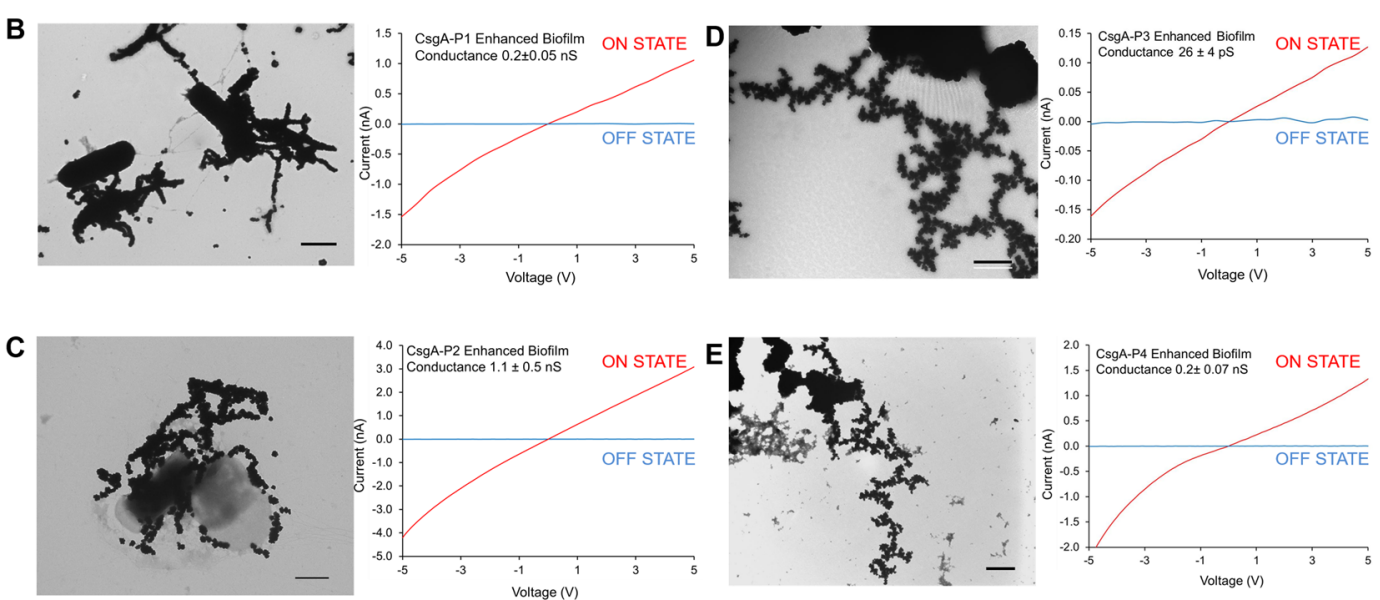

Figure 5. Conductive nanowire formation on cell-synthesized curli fibers with gold nucleation followed by gold enhancement. (A) Gold nanoparticles nucleated on peptide-displaying curli fibers were enhanced with gold ions to form nanowires. Red lines indicate conductance for cells induced for curli synthesis with aTc (ON STATE) while the blue lines indicate conductance for cells that were uninduced (OFF STATE). (B) CsgA-P1-based curli nanowires. (C) CsgA-P2-based curli nanowires had the greatest conductance out of the four peptide variants tested. (D) CsgAP3-based curli nanowires. (E) CsgA-P4-based curli nanowires. Scale bars on images represent $500 \mathrm{~nm}$. Conductance values are mean average measurements from three independent samples from three electrodes.

cellular communities can mediate electrical conductance without the need for preformed nanoparticles.

Our cell-based platform leverages the genetic tractability of living bacteria to control nanomaterials synthesis and implement inorganic-organic systems with electrical conductance. This work builds upon previous studies in which nanomaterials were synthesized and patterned using nonliving biomolecular templates. Phage particles displaying material-binding peptides have been widely used for such applications. ${ }^{13,32,33}$ Metalchelating viral templates have been used for engineering materials and devices such as batteries and solar cells. ${ }^{16}$ In addition, material-binding peptides fused to protein templates have been explored for biomedical and nanotechnology applications such as fluorescent labeling of biominerals, layerby-layer assembly of nanoparticles for device architectures, and oriented immobilization of protein on solid surfaces. ${ }^{20,34-37}$ In addition, bacteria have been shown to detoxify metallic ions in their environment by facilitating metallic nanoparticle synthesis $^{38-40}$ within the cytoplasm ${ }^{41}$ or in the surrounding environment. $^{42,43}$

Cell-based synthesis of protein nanomaterials can provide unique capabilities for materials science and nanotechnology via facile engineering of proteins and cells to mediate interfaces with inorganic materials and impart novel functionalities. For example, proteins can be designed to self-assemble into higherorder structures, ${ }^{44}$ and cells can be endowed with synthetic gene circuits to create temporal control over gene expression ${ }^{45}$ as well as self-organized patterns. ${ }^{46,47}$ In our previous work, ${ }^{5}$ we engineered synthetic gene circuits to control materials patterning and assembled presynthesized nanoparticles on curli fiber templates. Here, we built upon our prior efforts to functionalize curli amyloid fibers expressed by living bacterial communities to organize and synthesize gold nanoparticles, resulting in tunable electrical conductance. These features enabled the creation of conductive cellular populations mediated by inorganic nanomaterials. In future work, we may be able to incorporate recent advances in electrical and optogenetic gene regulation into this platform to achieve even greater spatiotemporal control of materials synthesis and patterning. Leveraging living cells to achieve nanomaterials synthesis may also enable novel interfaces to be established between other nonliving and living systems. ${ }^{48}$ Ultimately, we envision that hybrid biotic-abiotic materials and multifunctional biological materials based on engineered cellular populations could implement useful features such as environmental adaptation and self-repair.

\section{METHODS}

Genetic Engineering of Plasmids and Bacterial Cell Strains. To construct the E. coli MG1655 PRO $\Delta c s g A$ $o m p R 234$ strain used in this study, we sequentially created $E$. coli MG1655 PRO $\Delta c s g A:: a p h$, E. coli MG1655 PRO $\Delta c s g A$, and E. coli MG1655 PRO $\Delta c s g A o m p R 234$, as described by Chen et al. ${ }^{5}$ The PRO cassette includes high-level expression of the TetR and LacI transcription factors. ${ }^{12}$

The genetic parts and plasmids that were constructed in this study are listed in Table S1 and Table S4, respectively. The plasmids (Table S4) used in this study were constructed with standard molecular cloning techniques. ${ }^{49}$ Polymerase chain reaction (PCR), ligations, and restriction digestion reactions were carried out using a Bio-Rad S1000 Thermal Cycler with Dual 48/48 Fast Reaction Modules (Bio-Rad). PCR amplifications were carried out using Phusion PCR kits. To purify DNA, agarose gel extractions were carried out with QIAquick Gel Extraction Kits (Qiagen). Custom synthesized oligonucleotide primers were purchased from Integrated DNA 
Technologies (Coralville, IA). Restriction enzymes for digestions and T4 DNA ligase for ligation reactions were purchased from New England Biolabs.

The wild-type $\operatorname{csg} A$ gene and its variants were cloned downstream of a riboregulator system (Figure 1) in a mediumcopy-number pZA vector. The genes were placed under transcriptional regulation control by a $\mathrm{P}_{\text {LtetO }}$ promoter that can be induced by the addition of anhydrotetracycline (aTc) ${ }^{12}$ In addition, with the riboregulator system, translation of transcripts is repressed by a cis-repressing (cr) RNA to prevent translation of aberrant transcripts; this repression is lifted via the expression of a trans-activating RNA (taRNA) that is also under the regulation of $\mathrm{P}_{\mathrm{LtetO}}{ }^{11}$ To construct this vector, pZEAmpR-rr12- $\mathrm{P}_{\text {LtetO- }} f p$ was modified. ${ }^{50}$ First, the ampicillinresistance (AmpR) gene was replaced with the chloramphenicol-resistance (CmR) cassette from pZS-CmR-P $\mathrm{LlacO}_{-} g f p$ by using SacI-HF/AatII digestion and ligation to construct pZECmR-rr12-P $\mathrm{LtetO}_{-g f p}$. Next, the ColE1 origin of pZE-CmR-rr12-

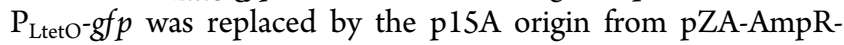
$\mathrm{P}_{\text {LtetO- }} g f p$ by using SacI-HF/AvrII digestion and ligation to create pZA-CmR-rr12-P $\mathrm{P}_{\text {LtetO }}-g f p$.

To clone $\operatorname{csg} A$ and the $\operatorname{csg} A$-peptide fusions $(\operatorname{csg} A-P 1, \operatorname{csg} A-P 2$, $\operatorname{csg} A-P 3$, and $\operatorname{csg} A-P 4)$, pZA-CmR-rr12- $\mathrm{P}_{\text {LtetO }} g f p$ was digested using MluI/KpnI digestion, and ligation was performed using T4 ligase. Primers used for each peptide insertion are given in Table S3 in the Supporting Information. The corresponding peptide sequences are given in Figure 3 and also in Table S1.

All ligations were transformed into E. coli strain DH5 $\alpha$ PRO using standard protocols. ${ }^{49}$ Isolated colonies were inoculated into LB-Miller medium (Fisher) using carbenicillin at $50 \mu \mathrm{g} /$ $\mathrm{mL}$ and/or chloramphenicol at $25 \mu \mathrm{g} / \mathrm{mL}$ where appropriate for plasmid maintenance. Plasmid DNA was extracted from liquid cultures using QIAprep Spin Miniprep Kits (Qiagen). Plasmid sequences were confirmed by restriction digestion and sequencing (Genewiz, Cambridge, MA). Sequence-verified plasmid constructs were transformed into E. coli MG1655 PRO $\Delta c s g A$ ompR234.

Induction of Curli Synthesis. Transformed cells were grown overnight in LB medium $\left(\sim 1 \times 10^{9}\right.$ cells $\left./ \mathrm{mL}\right)$, and 15 $\mu \mathrm{L}$ of overnight cultures was then inoculated into $2 \mathrm{~mL}$ of M63 minimal medium containing $0.2 \%$ glucose, $1 \mathrm{mM} \mathrm{MgSO}_{4}$, and $250 \mathrm{ng} / \mathrm{mL}$ aTc for induction of curli synthesis in 24-well plates at $30^{\circ} \mathrm{C}$. Following $48 \mathrm{~h}$ of incubation for CsgA-Wt and CsgA variants, planktonic cells and media were carefully drained, and wells were gently rinsed with phosphate-buffered saline (PBS) to remove loosely attached cells. Cells at the bottom of the wells were then removed by washing vigorously with PBS. The collected cells were further used for nanoparticle assembly experiments and nanowire growth experiments using gold enhancement, as described below.

In order to purify an increased amount of curli for biophysical characterization experiments, CsgA-expressing cells were also grown on YESCA (yeast extract-casamino acids) agar (10 g Casamino acids, $1 \mathrm{~g}$ yeast extract, $20 \mathrm{~g}$ agar per liter) with $250 \mathrm{ng} / \mathrm{mL}$ aTc for $72 \mathrm{~h}$ at $30{ }^{\circ} \mathrm{C}$. Following growth, curli-expressing cells were harvested with a spreader from the surface of the agar plates and resuspended in PBS. Curli fibers were then purified from these cell populations as previously described by Collinson et al. ${ }^{21}$

Assaying Amyloid Formation Efficiency of CsgA with Peptide Insertions. We used a 7xHis peptide tag to map out suitable insertion sites for heterologous peptides in CsgA. A plasmid expressing $\operatorname{csg} A$ under control of the $\mathrm{P}_{\mathrm{LlacO}}$ promoter was constructed from a backbone vector (pZS32G) with a pSC101 origin, chloramphenicol-resistance cassette, and $\mathrm{P}_{\text {LlacO. }}{ }^{12}$ The csgA gene was amplified from the E. coli genome by PCR, with flanking KpnI and MluI restriction sites encoded on primers. Different csgA genes containing DNA encoding the $7 \mathrm{xHis}$ peptide inserted in various positions were created by PCR with primers listed in the Supporting Information (Table S3). These genes were subsequently cloned into pZS32G at KpnI and MluI sites, where they replaced the $g f p$ gene. The resulting plasmids were transformed into a strain with the endogenous $\operatorname{csg} A$ gene removed (E. coli BW25113 $\Delta c s g A:: a p h$ from the Keio collection ${ }^{51}$ ) using standard techniques. ${ }^{49}$

Transformed cells were grown in LB with $25 \mu \mathrm{g} / \mathrm{mL}$ chloramphenicol overnight to saturation, and subsequently, 15 $\mu \mathrm{L}$ of each transformed strain was spotted onto YESCA agar with $25 \mu \mathrm{g} / \mathrm{mL}$ chloramphenicol and grown for $60 \mathrm{~h}$ at $30{ }^{\circ} \mathrm{C}$. As the host strain did not overexpress the LacI repressor protein, no IPTG was needed to induce expression of the various CsgA constructs from the $\mathrm{P}_{\text {LlacO }}$ promoter. The resulting colonies were resuspended in PBS. To quantitatively assay amyloid fiber formation, resuspended cell samples were incubated in Congo Red (CR) $(20 \mu \mathrm{g} / \mathrm{mL})$ for $5 \mathrm{~min}$ and centrifuged at high speed $(13,000 \mathrm{rcf})$. The unbound CR in the supernatant was assessed by measuring the sample absorbance at $480 \mathrm{~nm}$ using a microplate spectrophotometer ${ }^{52}$ and normalized to the $\mathrm{OD}_{600}$ of the cell suspension read prior to $\mathrm{CR}$ assay. Curli formation in Figure $2 \mathrm{~B}$ (in arbitrary units) was calculated by normalizing the absorption due to unbound Congo Red (A480) in the supernatant to the density of the initial cell suspension (A600), and inverting these units for ease of interpretation.

Gold-Nanoparticle Formation. Gold nanoparticles were formed on curli samples by the addition of $50 \mu \mathrm{M} \mathrm{HAuCl}_{4}$ (Sigma-Aldrich) and $10 \mu \mathrm{M}$ sodium citrate into cell suspensions containing curli-expressing cells which were previously induced with aTc and grown for $48 \mathrm{~h}$. Cells were incubated with $\mathrm{HAuCl}_{4}$ and sodium citrate for $72 \mathrm{~h}$ at room temperature.

Gold-Nanoparticle Assembly and Gold Enhancement. We used two different types of preformed gold nanoparticles (AuNPs) for the formation of gold nanowires on cellsynthesized curli fibers (Figure 4). NiNTA-coated $5 \mathrm{~nm}$ AuNPs (Nanoprobes, Inc.) and bare $5 \mathrm{~nm}$ AuNPs were assembled on curli samples in PBS by drop-casting. A drop (5 to $20 \mu \mathrm{L}$, higher volumes for larger surfaces) of $0.5 \mu \mathrm{M}$ AuNPs was added onto the cell-synthesized curli samples on the electrode surface. Bare gold nanoparticles are attached to the histidine tag on CsgA-P1 through metal-histidine coordination, as reported previously. ${ }^{53}$ Loosely bound nanoparticles were removed by rinsing with binding buffer (PBS with $0.487 \mathrm{M}$ $\mathrm{NaCl}, 80 \mathrm{mM}$ imidazole, and $0.2 \%$ (v/v) Tween 20). Gold enhancement was carried out on curli fibers after goldnanoparticle attachment to the curli or gold-nanoparticle growth on curli. Curli samples arrayed with gold particles were then subject to gold enhancement using the Gold Enhance Kit (Nanoprobes, Inc.). Subsequently, the samples were rinsed with distilled water. The same gold enhancement protocol was used for samples shown in Figure 4 and Figure 5.

Transmission Electron Microscopy (TEM) Imaging. TEM imaging was performed with a FEI Tecnai Spirit transmission electron microscope. Samples were prepared by floating 200-mesh Formvar/carbon-coated nickel TEM grids (Electron Microscopy Sciences) for $30 \mathrm{~s}$ on drops of solution 
containing curli. Following the adsorption of curli onto the TEM grids, rinsing was performed with binding buffer (PBS with $0.487 \mathrm{M} \mathrm{NaCl}, 80 \mathrm{mM}$ imidazole, and $0.2 \%$ (v/v) Tween 20) and distilled water. Thoroughly washed grids were placed face down on a drop of $1 \%(\mathrm{w} / \mathrm{v})$ uranyl acetate for negative staining. Excessive uranyl acetate was wicked off with Whatman filter paper, and grids were left to air-dry. Three independent grids were prepared for each curli sample. For the study of gold nanoparticles formed on curli fibers (Figure 3), 90 particles for CsgA-Wt, CsgA-P1, and CsgA-P3, and 80 particles for CsgA-P2 and CsgA-P4 were analyzed.

Scanning Electron Microscopy (SEM) Imaging. SEM imaging was performed using a FEI environmental SEM. Samples were prepared as follows: bacteria were grown in 24well plates in M63 minimal media supplemented with glucose $(2 \%, \mathrm{w} / \mathrm{v})$ and supplied with the inducer aTc $(250 \mathrm{ng} / \mathrm{mL})$ for 3 days in the case of biofilm expression. A silicon wafer was placed in each well, and medium was exchanged with the fixation solution ( $2 \%$ glutaraldehyde in $0.1 \mathrm{M}$ phosphate buffer) and incubated overnight at $4{ }^{\circ} \mathrm{C}$. A series of ethanol solutions with increasing alcohol content (40\%, 70\%, 96\%, 100\%) was used to remove fixation reagent and excess water from the biofilm adhered to the silicon wafer (plate was shaken for 20 min between each wash step). Wafers were air-dried for an hour, and gold-palladium film was sputter coated on top of the biofilm (film thickness $5 \mathrm{~nm}$ ). E-beam energy (5 keV) was applied to capture SEM images.

Quartz Crystal Microbalance. Characterization of goldbinding interactions with purified CsgA-Wt curli fibers and curli fiber variants was carried out using a four channel QCM-D (QSense, Biolin Scientific, Sweden). The QCM-D was equipped with a peristaltic pump, and sample solutions were moved to the chamber, where the gold-coated sensors were located, by pumping with a flow rate of $100 \mu \mathrm{L} \mathrm{min}{ }^{-1}$. Purified curli samples were prepared from agar-grown samples suspended in PBS. Each sample was sonicated in a ultrasonic bath to ensure a well-suspended curli solution and to prevent clumping. Three independent replicates were prepared for each of the curli samples.

Before running QCM-D with curli samples, the sensor was equilibrated with PBS by flowing the buffer until a stable baseline was reached. Curli samples were then flowed through the flow cell until equilibrium for the adsorption of curli onto the sensor surface was reached. Concentrations for each curli sample are given in Figure S1. Following curli binding onto the sensor surface, unbound and nonspecifically bound curli fibers were removed from the surface by extensive washing of the sensor with PBS. Collected data was analyzed using a single Langmuir adsorption model fitted to the data points using an iterative least-squares fitting method. ${ }^{22}$

Circular Dichroism Spectroscopy. The secondarystructure motifs of CsgA-Wt fibers and peptide-displaying CsgA fibers were analyzed using an Aviv Model 202 Circular Dichroism spectrometer with a quartz cuvette with $1 \mathrm{~mm}$ path length. Purified curli fibers from agar-grown strains were prepared in PBS and sonicated for 5 min before measurements were taken. Each sample was prepared independently in duplicate. Each cuvette was measured three times, and measurements were overlaid and averaged by the software. Collected data was analyzed using the CDPro software package as described previously ${ }^{54}$ and used to generate a linear model. The model includes the percentage of each secondary-structure element, which was determined based on reference curves and secondary-structure data from a library of proteins. The details of the modeling can be found in previous work by Reed et al. and Greenfield et al. ${ }^{55,56}$

Conductance Measurements. Conductance of cellular populations was measured using interdigitated electrodes (IDEs) that were prepared using a shadowing mask (TechEtch). To prepare the electrodes, $\sim 200 \mathrm{~nm}$ of gold was sputtered (Desk II Sputter Coater, Denton Vacuum) through the shadowing mask onto Thermanox coverslips. Using a Keithley 4200 parameter analyzer with a two-point probe, empty electrodes were first tested for short circuits before usage. Electrodes were then cleaned with solvent in the following order: isopropanol/ethanol/deionized water. Clean IDEs were placed in 24-well polystyrene wells. M63 media with $0.2 \%$ glucose and $1 \mathrm{mM} \mathrm{MgSO}$ were added into the wells, along with $250 \mathrm{ng} / \mathrm{mL}$ aTc. Cells were then inoculated at $\sim 1 \times$ $10^{9}$ cells $/ \mathrm{mL}$ and grown for $24 \mathrm{~h}$ at $30{ }^{\circ} \mathrm{C}$ without shaking. Control experiments were carried out by growing cells under the same conditions in the absence of aTc induction. Conductance measurements were carried out after gold enhancement of the preformed gold nanoparticles attached to curli fibers (Figure 4) and after gold enhancement of the gold nanoparticles nucleated on curli fibers using the methods described above.

\section{ASSOCIATED CONTENT}

\section{Supporting Information}

The Supporting Information is available free of charge on the ACS Publications website at DOI: 10.1021/acssynbio.6b00166.

DNA sequences and amino acid sequences of the corresponding genetic constructs, raw data for QCM-D experiments and circular dichroism data for CsgA proteins, details of the experimental methods (PDF)

\section{AUTHOR INFORMATION}

\section{Corresponding Author}

*E-mail: timlu@mit.edu.

\section{Author Contributions}

U.O.S.S. and T.K.L. conceived the experiments, U.O.S. S., A.Y.C., and R.J.C performed the experiments, and all authors analyzed the data, discussed results, and wrote the manuscript. Notes

The authors declare no competing financial interest.

\section{ACKNOWLEDGMENTS}

We thank J. J. Collins (Biomedical Engineering, Boston University) for riboregulator plasmids and C. Dorel (Biosciences Department, INSA Lyon) for E. coli MG1655 ompR234. This work was supported by the Army Research Office and the Office of Naval Research. This work was also supported in part by the MRSEC Program of the National Science Foundation under award number DMR-0819762. A.Y.C. acknowledges graduate research support from the Hertz Foundation, the Department of Defense, and NIH Medical Scientist Training Program grant T32GM007753. T.K.L. also acknowledges support from the Presidential Early Career Award for Scientists and Engineers and the NIH New Innovator Award (1DP2OD008435). U.O.S.S. acknowledges the Turkish Academy of Sciences Distinguished Young Scientist Award (TUBA-GEBIP). U.O.S.S. thanks Tolga Tarkan Olmez for his help with SEM imaging. 


\section{REFERENCES}

(1) Chapman, M. R., Robinson, L. S., Pinkner, J. S., Roth, R., Heuser, J., Hammar, M., Normark, S., and Hultgren, S. J. (2002) Role of Escherichia coli curli operons in directing amyloid fiber formation. Science 295, 851-855.

(2) Kirsten, A., Herzberg, M., Voigt, A., Seravalli, J., Grass, G., Scherer, J., and Nies, D. H. (2011) Contributions of five secondary metal uptake systems to metal homeostasis of Cupriavidus metallidurans CH34. J. Bacteriol. 193, 4652-4663.

(3) Taylor, J. D., Zhou, Y., Salgado, P. S., Patwardhan, A., McGuffie, M., Pape, T., Grabe, G., Ashman, E., Constable, S. C., Simpson, P. J., Lee, W. C., Cota, E., Chapman, M. R., and Matthews, S. J. (2011) Atomic resolution insights into curli fiber biogenesis. Structure 19, 1307-1316.

(4) Dueholm, M. S., Albertsen, M., Otzen, D., and Nielsen, P. H. (2012) Curli functional amyloid systems are phylogenetically widespread and display large diversity in operon and protein structure. PLoS One 7, e51274.

(5) Chen, A. Y., Deng, Z., Billings, A. N., Seker, U. O. S., Lu Michelle, Y., Citorik, R. J., Zakeri, B., and Lu, T. K. (2014) Synthesis and patterning of tunable multiscale materials with engineered cells. Nat. Mater. 13, 515-523.

(6) Barnhart, M. M., and Chapman, M. R. (2006) Curli biogenesis and function, in Annu. Rev. Microbiol., pp 131-147, Annual Reviews, Palo Alto.

(7) Shu, Q., Crick, S. L., Pinkner, J. S., Ford, B., Hultgren, S. J., and Frieden, C. (2012) The E. coli CsgB nucleator of curli assembles to beta-sheet oligomers that alter the CsgA fibrillization mechanism. Proc. Natl. Acad. Sci. U. S. A. 109, 6502-6507.

(8) Wang, X., Zhou, Y. Z., Ren, J. J., Hammer, N. D., and Chapman, M. R. (2010) Gatekeeper residues in the major curlin subunit modulate bacterial amyloid fiber biogenesis. Proc. Natl. Acad. Sci. U. S. A. $107,163-168$.

(9) Prigent-Combaret, C., Brombacher, E., Vidal, O., Ambert, A., Lejeune, P., Landini, P., and Dorel, C. (2001) Complex regulatory network controls initial adhesion and biofilm formation in Escherichia coli via regulation of the $\operatorname{csgD}$ gene. J. Bacteriol. 183, 7213-7223.

(10) Boehm, A., and Vogel, J. (2012) The csgD mRNA as a hub for signal integration via multiple small RNAs. Mol. Microbiol. 84, 1-5.

(11) Isaacs, F. J., Dwyer, D. J., Ding, C., Pervouchine, D. D., Cantor, C. R., and Collins, J. J. (2004) Engineered riboregulators enable posttranscriptional control of gene expression. Nat. Biotechnol. 22, 841847.

(12) Lutz, R, and Bujard, H. (1997) Independent and tight regulation of transcriptional units in Escherichia coli via the LacR/O, the TetR/O and AraC/I-1-I-2 regulatory elements. Nucleic Acids Res. $25,1203-1210$.

(13) Seker, U. O. S., and Demir, H. V. (2011) Material Binding Peptides for Nanotechnology. Molecules 16, 1426-1451.

(14) Sarikaya, M., Tamerler, C., Jen, A. K., Schulten, K., and Baneyx, F. (2003) Molecular biomimetics: nanotechnology through biology. Nat. Mater. 2, 577-585.

(15) Tamerler, C., Khatayevich, D., Gungormus, M., Kacar, T., Oren, E. E., Hnilova, M., and Sarikaya, M. (2010) Molecular Biomimetics: GEPI-Based Biological Routes to Technology. Biopolymers 94, 78-94.

(16) Lee, Y. J., Yi, H., Kim, W. J., Kang, K., Yun, D. S., Strano, M. S., Ceder, G., and Belcher, A. M. (2009) Fabricating genetically engineered high-power lithium-ion batteries using multiple virus genes. Science 324, 1051-1055.

(17) Nam, K. T., Kim, D. W., Yoo, P. J., Chiang, C. Y., Meethong, N., Hammond, P. T., Chiang, Y. M., and Belcher, A. M. (2006) Virusenabled synthesis and assembly of nanowires for lithium ion battery electrodes. Science 312, 885-888.

(18) Slocik, J. M., Stone, M. O., and Naik, R. R. (2005) Synthesis of gold nanoparticles using multifunctional peptides. Small 1, 10481052 .

(19) Kim, J., Rheem, Y., Yoo, B., Chong, Y., Bozhilov, K. N., Kim, D., Sadowsky, M. J., Hur, H. G., and Myung, N. V. (2010) Peptide- mediated shape- and size-tunable synthesis of gold nanostructures. Acta Biomater. 6, 2681-2689.

(20) Yuca, E., Karatas, A. Y., Seker, U. O. S., Gungormus, M., DinlerDoganay, G., Sarikaya, M., and Tamerler, C. (2011) In Vitro Labeling of Hydroxyapatite Minerals by an Engineered Protein. Biotechnol. Bioeng. 108, 1021-1030.

(21) Collinson, S. K., Emody, L., Muller, K. H., Trust, T. J., and Kay, W. W. (1991) Purification and Characterization of Thin, Aggregative Fimbriae from Salmonella-Enteritidis. J. Bacteriol. 173, 4773-4781.

(22) Seker, U. O. S., Wilson, B., Sahin, D., Tamerler, C., and Sarikaya, M. (2009) Quantitative Affinity of Genetically Engineered Repeating Polypeptides to Inorganic Surfaces. Biomacromolecules 10, 250-257.

(23) Seker, U. O. S., Wilson, B., Dincer, S., Kim, I. W., Oren, E. E., Evans, J. S., Tamerler, C., and Sarikaya, M. (2007) Adsorption behavior of linear and cyclic genetically engineered platinum binding peptides. Langmuir 23, 7895-7900.

(24) Chen, H., Su, X., Neoh, K. G., and Choe, W. S. (2009) Contextdependent adsorption behavior of cyclic and linear peptides on metal oxide surfaces. Langmuir 25, 1588-1593.

(25) Rambaran, R. N., and Serpell, L. C. (2008) Amyloid fibrils Abnormal protein assembly. Prion 2, 112-117.

(26) Polte, J., Erler, R., Thunemann, A. F., Sokolov, S., Ahner, T. T., Rademann, K., Emmerling, F., and Kraehnert, R. (2010) Nucleation and growth of gold nanoparticles studied via in situ small angle $\mathrm{X}$-ray scattering at millisecond time resolution. ACS Nano 4, 1076-1082.

(27) Peelle, B. R., Krauland, E. M., Wittrup, K. D., and Belcher, A. M. (2005) Design criteria for engineering inorganic material-specific peptides. Langmuir 21, 6929-6933.

(28) Wei, H., Wang, Z., Zhang, J., House, S., Gao, Y. G., Yang, L., Robinson, H., Tan, L. H., Xing, H., Hou, C., Robertson, I. M., Zuo, J. M., and Lu, Y. (2011) Time-dependent, protein-directed growth of gold nanoparticles within a single crystal of lysozyme. Nat. Nanotechnol. 6, 93-97.

(29) Payne, S., Li, B., Cao, Y., Schaeffer, D., Ryser, M. D., and You, L. Temporal control of self-organized pattern formation without morphogen gradients in bacteria. Mol. Syst. Biol. 2013, 9, 10.1038/ $\mathrm{msb} .2013 .55$.

(30) Lovley, D. R. (2012) Electromicrobiology. In: Gottesman, S., Harwood, C. S., and Schneewind, O., editors. Annu. Rev. Microbiol. 66 , 391-409.

(31) TerAvest, M. A., Li, Z. J., and Angenent, L. T. (2011) Bacteriabased biocomputing with Cellular Computing Circuits to sense, decide, signal, and act. Energy Environ. Sci. 4, 4907-4916.

(32) Sarikaya, M. (1999) Biomimetics: materials fabrication through biology. Proc. Natl. Acad. Sci. U. S. A. 96, 14183-14185.

(33) Flynn, C. E., Lee, S. W., Peelle, B. R., and Belcher, A. M. (2003) Viruses as vehicles for growth, organization and assembly of materials. Acta Mater. 51, 5867-5880.

(34) Sano, K., Sasaki, H., and Shiba, K. (2006) Utilization of the pleiotropy of a peptidic aptamer to fabricate heterogeneous nanodotcontaining multilayer nanostructures. J. Am. Chem. Soc. 128, 17171722.

(35) Krauland, E. M., Peelle, B. R., Wittrup, K. D., and Belcher, A. M. (2007) Peptide tags for enhanced cellular and protein adhesion to single-crystal line sapphire. Biotechnol. Bioeng. 97, 1009-1020.

(36) Kacar, T., Zin, M. T., So, C., Wilson, B., Ma, H., Gul-Karaguler, N., Jen, A. K. Y., Sarikaya, M., and Tamerler, C. (2009) Directed SelfImmobilization of Alkaline Phosphatase on Micro-Patterned Substrates Via Genetically Fused Metal-Binding Peptide. Biotechnol. Bioeng. 103, 696-705.

(37) Koch, D., Chan, A. C., Murphy, M. E., Lilie, H., Grass, G., and Nies, D. H. (2011) Characterization of a dipartite iron uptake system from uropathogenic Escherichia coli strain F11. J. Biol. Chem. 286, 25317-25330.

(38) Chaturvedi, K. S., Hung, C. S., Crowley, J. R., Stapleton, A. E., and Henderson, J. P. (2012) The siderophore yersiniabactin binds copper to protect pathogens during infection. Nat. Chem. Biol. 8, 731736. 
(39) Kim, H. J., Graham, D. W., DiSpirito, A. A., Alterman, M. A., Galeva, N., Larive, C. K., Asunskis, D., and Sherwood, P. M. A. (2004) Methanobactin, a copper-acquisition compound from methaneoxidizing bacteria. Science 305, 1612-1615.

(40) Johnston, C. W., Wyatt, M. A., Li, X., Ibrahim, A., Shuster, J., Southam, G., and Magarvey, N. A. (2013) Gold biomineralization by a metallophore from a gold-associated microbe. Nat. Chem. Biol. 9, 241243.

(41) Klaus, T., Joerger, R., Olsson, E., and Granqvist, C. G. (1999) Silver-based crystalline nanoparticles, microbially fabricated. Proc. Natl. Acad. Sci. U. S. A. 96, 13611-13614.

(42) Ramanathan, R., O'Mullane, A. P., Parikh, R. Y., Smooker, P. M., Bhargava, S. K., and Bansal, V. (2011) Bacterial kinetics-controlled shape-directed biosynthesis of silver nanoplates using Morganella psychrotolerans. Langmuir 27, 714-719.

(43) Parikh, R. Y., Singh, S., Prasad, B. L., Patole, M. S., Sastry, M., and Shouche, Y. S. (2008) Extracellular synthesis of crystalline silver nanoparticles and molecular evidence of silver resistance from Morganella sp.: towards understanding biochemical synthesis mechanism. ChemBioChem 9, 1415-1422.

(44) Grass, G., Otto, M., Fricke, B., Haney, C. J., Rensing, C., Nies, D. H., and Munkelt, D. (2005) FieF (YiiP) from Escherichia coli mediates decreased cellular accumulation of iron and relieves iron stress. Arch. Microbiol. 183, 9-18.

(45) Ellis, T., Wang, X., and Collins, J. J. (2009) Diversity-based, model-guided construction of synthetic gene networks with predicted functions. Nat. Biotechnol. 27, 465-471.

(46) Payne, S., Li, B., Cao, Y., Schaeffer, D., Ryser, M. D., and You, L. (2013) Temporal control of self-organized pattern formation without morphogen gradients in bacteria. Mol. Syst. Biol. 9, 697.

(47) Jang, S. S., Oishi, K. T., Egbert, R. G., and Klavins, E. (2012) Specification and simulation of synthetic multicelled behaviors. ACS Synth. Biol. 1, 365-374.

(48) Leduc, P. R., Wong, M. S., Ferreira, P. M., Groff, R. E., Haslinger, K., Koonce, M. P., Lee, W. Y., Love, J. C., McCammon, J. A., Monteiro-Riviere, N. A., Rotello, V. M., Rubloff, G. W., Westervelt, R., and Yoda, M. (2007) Towards an in vivo biologically inspired nanofactory. Nat. Nanotechnol. 2, 3-7.

(49) Nies, D. H. (2003) Efflux-mediated heavy metal resistance in prokaryotes. FEMS Microbiol Rev. 27, 313-339.

(50) Chang, L. Y., Lunt, R. R., Brown, P. R., Bulovic, V., and Bawendi, M. G. (2013) Low-temperature solution-processed solar cells based on $\mathrm{PbS}$ colloidal quantum dot/CdS heterojunctions. Nano Lett. 13, 994-999.

(51) Baba, T., Ara, T., Hasegawa, M., Takai, Y., Okumura, Y., Baba, M., Datsenko, K. A., Tomita, M., Wanner, B. L., and Mori, H. (2006) Construction of Escherichia coli K-12 in-frame, single-gene knockout mutants: the Keio collection, Mol. Syst. Biol. 2.10.1038/msb4100050

(52) Reichhardt, C., Jacobson, A. N., Maher, M. C., Uang, J., McCrate, O. A., Eckart, M., and Cegelski, L. (2015) Congo Red Interactions with Curli-Producing E. coli and Native Curli Amyloid Fibers, PLoS One 10.10.1371/journal.pone.0140388

(53) Aldeek, F., Safi, M., Zhan, N. Q., Palui, G., and Mattoussi, H.

(2013) Understanding the Self-Assembly of Proteins onto Gold Nanoparticles and Quantum Dots Driven by Metal-Histidine Coordination. ACS Nano 7, 10197-10210.

(54) Seker, U. O. S., Ozel, T., and Demir, H. V. (2011) PeptideMediated Constructs of Quantum Dot Nanocomposites for Enzymatic Control of Nonradiative Energy Transfer. Nano Lett. 11, 1530-1539.

(55) Reed, J., and Reed, T. A. (1997) A set of constructed type spectra for the practical estimation of peptide secondary structure from circular dichroism. Anal. Biochem. 254, 36-40.

(56) Greenfield, N. J. (2006) Using circular dichroism spectra to estimate protein secondary structure. Nat. Protoc 1, 2876-2890. 